\title{
Comorbilidad psicopatológica en consumidores de cocaína en tratamiento ambulatorio
}

\author{
Psychopathological comorbidity in cocaine \\ users in outpatient treatment
}

\begin{abstract}
Pedro Araos*; Esperanza Vergara-Moragues**; María Pedraz*; Francisco J. Pavón*; Rafael Campos Cloute***; Montserrat Calado*****; Juan Jesús Ruiz*****; Nuria García-Marchena*; Isolde Gornemann*; Marta Torrens******; Fernando Rodríguez de Fonseca*

*Unidad de Gestión Cínica de Salud Mental, Hospital Regional Universitario Carlos Haya-Instituto IBIMA, Málaga;

**Universidad Internacional de la Rioja (UNIR), Logroño; ***Centro Comarcal de Drogodependencias, Mijas Costa; ****Servicio de Neurología, Hospital Virgen Macarena, Sevilla; *****Centro de Tratamiento Ambulatorio, Málaga; ******Human Pharmacology and Clinical Neurosciences Research Group, Institut Hospital del Mar d'Investigacions Mèdiques (IMIM), Barcelona.
\end{abstract}

\section{Resumen}

La adicción a cocaína es un problema de salud creciente y entre sus complicaciones destaca la elevada prevalencia de comorbilidad psicopatológica. La detección temprana de los trastornos psicopatológicos asociados al consumo de cocaína es necesaria para optimizar la asistencia sanitaria y mejorar el pronóstico. El objetivo principal de este estudio es estimar la prevalencia y características de la comorbilidad psicopatológica en una población de sujetos que solicitan atención por uso de cocaína en tratamiento ambulatorio. Se reclutaron 110 consumidores de cocaína por vía nasal evaluados con la entrevista diagnóstica semiestructurada PRISM (entrevista de investigación psiquiátrica para trastornos mentales y por sustancias), que diferencia los trastornos mentales primarios de los inducidos por la droga. Esta población tuvo un $86,4 \%$ de hombres y una edad media de 36,5 años. Presentó un uso patológico de cocaína medio de 7 años, y la presencia de psicopatología se asoció a un mayor número de criterios de dependencia de cocaína según el manual DSM-IVTR (manual diagnóstico y estadístico de los trastornos mentales, $4^{a}$ edición revisada). La prevalencia de comorbilidad psicopatológica encontrada a lo largo de la vida fue del $61,8 \%$, destacando los trastornos del estado de ánimo (34,5\%), seguidos de los trastornos de ansiedad (22,7\%) y de los trastornos psicóticos (15,5\%). Un $20 \%$ presentó trastorno de personalidad antisocial y un $21 \%$ trastorno límite de la personalidad. Entre los trastornos del estado de ánimo y psicóticos fueron más frecuentes los inducidos, mientras que en los trastornos de ansiedad los primarios fueron más prevalentes. Palabras clave: comorbilidad, psicopatología, cocaína, abuso, dependencia.

\section{Abstract}

Cocaine addiction is a growing health problem and among its complications highlights the high prevalence of mental disorders co-occurring with abuse and dependence. This psychopathological comorbidity varies according to the time of consumption and the age of the patient. Early detection of psychopathological disorders associated with drug consumption is necessary to optimize health care and to improve the prognosis. The main aim of the present study was to estimate the prevalence and characteristics of psychopathological comorbidity in a population of subjects seeking outpatient treatment for cocaine use. We recruited 110 subjects using cocaine by nasal insufflations evaluated with the PRISM (Psychiatric Research Interview for Substance and Mental Disorders), a semi-structured diagnostic interview that differentiates primary mental disorders from those induced by the drug. This population presented $86.4 \%$ male and had a mean age of 36.5. They displayed a pathological use of cocaine of 7 years and the presence of psychopathology was associated with a higher number of DSM-IV-TR (Diagnostic and Statistical Manual of Mental DisordersIV-TR) criteria for substance dependence. The lifetime prevalence of some psychopathological comorbidity was $61.8 \%$, highlighting mood disorders $(34.5 \%)$, followed by anxiety disorders $(22.7 \%)$ and psychotic disorders $(15.5 \%)$. About $20 \%$ showed antisocial personality disorder and $21 \%$ borderline personality disorder. From among mood and psychotic disorders, the induced disorders were more frequent, while the primary disorders were more prevalent in anxiety. Key words: comorbidity, psychopathology, cocaine, abuse, dependence.

Recibido: marzo 2013; Aceptado: julio 2013

Enviar correspondencia a:

Dr. Fernando Rodriguez de Fonseca. INSTITUTO IBIMA, Hospital Carlos Haya de Málaga. Unidad Clínica de Gestión de Salud Mental.

Pabellón de Gobierno Sótano. Avenida de Carlos Haya 82, Málaga 29010. Tf +34669426548. E-mail: fernando.rodriguez@fundacionimabis.org 
$\mathrm{E}$ l consumo de cocaína es un problema de salud muy importante en las sociedades actuales, con una alta prevalencia en edades tempranas y un fuerte impacto social, sanitario y económico (Office of National Drug Control Policy (ONDCP), 2012; Rivera, Casal, Currais y Rungo, 2012).

La comorbilidad psicopatológica, es decir, la coexistencia en una misma persona de un trastorno por uso de sustancia (TUS) y de otras psicopatologías, constituye un problema serio de salud (Goldsmith, 1999). Estos pacientes con comorbilidad psicopatológica conforman un grupo de riesgo desde una visión clínica y social. Frecuentan en mayor medida los servicios hospitalarios (Rufles, 2009), tienen mayores tasas de suicidios (Fiedler et al., 2012) y tienen valores de respuestas al tratamiento peores que pacientes solo con TUS (Karila, Petit, Lowenstein y Reynaud, 2012). Por otra parte, desde un punto de vista social son pacientes con mayor carga de conflictos a nivel laboral, judicial y de inserción social (Karila et al. 2013).

En un gran número de estudios, se muestra la relación del uso de cocaína con la elevada prevalencia de comorbilidad psicopatológica (Gual, 2007; Pavarin, 2006; Torrens, Gilchrist y Domingo-Salvany, 2011). En esta línea, en nuestro contexto, se han realizado investigaciones que relacionan la presencia de una alta comorbilidad psicopatológica en consumidores de cocaína. La prevalencia es elevada y se sitúa entre un 30 y un $65 \%$ tanto en población clínica como no clínica (Arias et al., 2012; Herrero, Domingo-Salvany, Torrens, Brugal y the ITINERE investigator, 2007; Herrero, Domingo-Salvany, Torrens, Brugal, Gutierrez y the ITINERE investigator, 2008; Tortajada et al., 2012; Vergara-Moragues et al. 2012). La variabilidad observada entre estas prevalencias podría atribuirse, por un lado a las variaciones en las tendencias de la población debido a la disponibilidad de la sustancia en cada momento y, por otro, a las diferencias en el proceso de muestreo. Es decir, los resultados difieren en función de la composición de las series (tipos de población, vías de administración, etc.) y de la metodología empleada (instrumentos de medida, tiempo transcurrido desde la abstinencia, entrenamiento en el diagnóstico psicopatológico de los entrevistadores, etc.). En cualquier caso, y considerando los datos de forma global, podemos concluir que la prevalencia de comorbilidad psicopatológica entre pacientes consumidores de cocaína, es muy elevada (Rounsaville, 2004; Schuckit, 2006; Weiser et al., 2003).

Por lo tanto, es importante conocer qué tipo de trastorno presenta cada persona y las características del mismo. Esta distinción sería útil para realizar un intervención adecuada y mejorar el pronóstico (Schuckit, 2006). En suma, el estudio de los trastornos concomitantes con el TUS, en este caso cocaína, tiene relevantes implicaciones teóricas y prácticas, tanto de tipo etiológico (construcción de modelos explicativos), como diagnóstico (creación de subgrupos específicos) y pronóstico (los sujetos con trastornos añadidos al TUS tienen más complicaciones durante en tratamiento que los que sólo presentan un TUS). Por todo esto, se señala la necesidad de seguir investigando, las consecuencias que tiene tratar trastornos mentales asociados a la evolución de trastornos adictivos (Vergara-Moragues et al., 2012).

Además de todo lo anterior, según lo informado por el Observatorio Europeo de las Drogas y las Toxicomanías (OEDT) en su informe anual de 2012, el 10,2\% de la población española entre 15 y 64 años ha consumido cocaína a lo largo de la vida, el 2,7\% lo ha hecho durante el último año y el 1,3\% durante el último mes. El consumo de cocaína entre adultos jóvenes en población española (de 15 a 34 años) refleja cifras aún superiores, el 13,6\% ha consumido cocaína a lo largo de la vida, el 4,4\% lo ha hecho el último año y el 2,0\% durante el último mes. Por tanto, España es el país de la Unión Europea donde más cocaína se ha consumido en estos últimos años, duplicando en muchos casos la media comunitaria.

Por tal motivo, es importante poner énfasis en las diferentes fases del consumo y en la escalada hacia el desarrollo de abuso y dependencia. Se estima que el 5-6\% de los usuarios que consumen cocaína desarrollarán una dependencia en el primer año de utilización de la sustancia, marcadamente superior al $1 \%$ relacionado con el cannabis. (Wagner y Anthony, 2006).

De esta manera, desde el primer contacto con la sustancia, se pueden distinguir diferentes etapas de consumo: uso, abuso y dependencia. Así considerados, el uso, y el posterior desarrollo de abuso y dependencia de cocaína, emergen antes y de forma más explosiva que el de otras drogas como alcohol y cannabis (Wagner y Anthony, 2006). En esta línea, la media de edad para el desarrollo de dependencia comprende entre los 23-25 años de edad y la estimación del primer consumo no abusivo entre los 21-22 años de edad. (Wagner y Anthony, 2006; OEDT, 2012).

Por otro lado, nos parece importante comparar la psicopatología de distintos estudios de nuestra comunidad autónoma (Andalucía). Las muestras consideradas fueron de población general (PG; N=1600) (Gornemann, 2002), nuestro estudio en tratamiento ambulatorio CTA $(\mathrm{N}=110) \mathrm{y}$, por último, otro trabajo realizado en comunidades terapéuticas públicas de Andalucía (CT; N=227) (Vergara et al. 2012).

La detección temprana de comorbilidad psicopatológica en centros terapéuticos ambulatorios se tornaría prioritaria con la finalidad de llevar un adecuado proceso de asistencia sanitaria en esta población y minimizar la falta de adherencia a los tratamientos y las frecuentes recaídas que ensombrecen el pronóstico de estos pacientes (Dutra et al., 2008). Por todos estos motivos, el objetivo principal de este estudio es estimar la prevalencia y características de la comorbilidad psicopatológica en una población de sujetos que han solicitado atención ambulatoria por consumo de cocaína. Asimismo, se compara la psicopatología de tres distintas poblaciones de nuestro entorno. 


\section{Material y Métodos}

\section{Diseño del estudio}

Se ha realizado un estudio observacional y descriptivo, con un componente retrospectivo.

\section{Población de estudio}

El estudio se realizó con una muestra total de 110 sujetos consumidores de cocaína por vía intranasal, que solicitaron atención en distintos centros de tratamiento ambulatorio (CTA) de drogodependencias de la provincia de Málaga [CTA-Mijas-Costa, centro concertado Alternativa 2-Fuengirola, CTA- Málaga (Carretera de Cádiz) y CTA-Vélez-Málaga]. Estos centros son coordinados por la dirección de la Diputación de Málaga y están especializados en la atención integral de los problemas derivados de las drogas y sus adicciones. Los objetivos generales de estos dispositivos son atender las demandas como centro especializado en la atención primaria colaborando con las instituciones socio-sanitarias para la rehabilitación y reinserción de las personas afectadas.

Además, estos recursos son la puerta de entrada al circuito terapéutico de la red asistencial de drogodependencias de la Junta de Andalucía. Por ello, es el canal de acceso al resto de los dispositivos (unidades de desintoxicación hospitalaria, comunidades terapéuticas, viviendas de apoyo al tratamiento, viviendas de apoyo a la reinserción, centros de día y centros de encuentros y acogidas) y programas disponibles en la comunidad andaluza.

Tomando como referencia el trabajo de Herrero y colaboradores (Herrero et al., 2007), la prevalencia de comorbilidad psicopatológica en consumidores de cocaína es del $42 \%$ aproximadamente. Para conseguir una precisión del $9 \%$ en la estimación de una proporción mediante un intervalo de confianza asintótico normal al $95 \%$ bilateral, asumiendo que la proporción es del 42\%, será necesario incluir para este estudio una muestra de 110 sujetos.

Se incluyeron para nuestro estudio pacientes que iniciaron tratamientos de media y larga duración y, que tuvieran un periodo de abstinencia controlada de al menos 30 días al momento de la entrevista.

Los criterios de inclusión que se han tenido en cuenta son: personas mayores de 18 años, consumidores de cocaína (tanto sujetos con consumo de cocaína que no cumplen criterios de abuso y/ o dependencia según DSM-IV-TR, como sujetos que sí cumplen estos criterios) y que hayan firmado el consentimiento informado. Cumpliendo los anteriores, los criterios de exclusión son: presencia de alteraciones cognitivas o sintomatología psicótica que imposibiliten la aplicación de los instrumentos diagnósticos y el rechazo a participar en el estudio. Los aspectos éticos de esta investigación fueron aprobados por el Comité de Ética e Investigación Clínica del Hospital Regional Universitario Carlos Haya de Málaga, en el ámbito de un proyecto financiado por el Plan Nacional Sobre Drogas y respetando los principio éticos para la investigación médica sobre sujetos humanos adoptados en la Declaración de Helsinki (Asociación Médica Mundial).

El proceso de evaluación fue realizado por un psicólogo clínico con formación especializada y acreditada en evaluación psicopatológica.

\section{Instrumento de medida}

La entrevista de diagnóstico PRISM (Psychiatric Research Interview for Substance and Mental Diseases) fue utilizada para valorar los trastornos psicopatológicos y por sustancias. PRISM es una entrevista clínica semiestructurada diseñada para resolver los problemas de diagnóstico en personas con un consumo elevado de sustancias y/o alcohol, evaluando en un primer módulo preguntas del historial de consumo del sujeto, arrojando diagnósticos de abuso y dependencia tanto en el último año como en el pasado. Además, evalúa 20 trastornos del Eje I y los dos trastornos del Eje II más prevalentes en esta población: el trastorno límite de la personalidad y el trastorno antisocial de la personalidad. Los diagnósticos se realizan en dos marcos temporales de evaluación. Por un lado, la entrevista valora los trastornos actuales, es decir, los que están presentes durante el último año. Por el otro, evalúa los trastornos previos, es decir, los definidos con anterioridad al último año. De manera general, la prevalencia diagnóstica presentada por un sujeto "a lo largo de la vida”, incluiría la totalidad de los diagnósticos enunciados con anterioridad observados en ambos periodos temporales, es decir, los actuales más los previos.

Una de las características más importantes de este instrumento es que permite diferenciar los trastornos mentales primarios o independientes de los trastornos inducidos por sustancias, así como los síntomas esperados del efecto de la intoxicación y la abstinencia. El criterio establecido por la PRISM para que un trastorno psicopatológico pueda ser considerado inducido por la sustancia, es que debe ocurrir en el contexto de un consumo patológico de la sustancia, en cualquiera de estas dos situaciones: a) intoxicación crónica, es decir, un consumo de cuatro o más días a la semana durante un mes; b) atracones, durante un periodo de consumo de tres días continuados. Para diferenciar los síntomas psicopatológicos inducidos de los esperados durante la intoxicación o la abstinencia, se debe determinar si hubo un cambio brusco en el patrón de consumo. (Hasin et al., 1996; Torrens, Serrano, Astals, Perez-Domínguez y Martin-Santos, 2004).

Esta entrevista presenta buena fiabilidad test-retest, validez y fiabilidad inter-examinadores (coeficiente Kappa oscila entre 0,66 y 1,00) (Morgello et al., 2006).

\section{Variables de estudio}

Comorbilidad psicopatológica y prevalencia de trastornos primarios e inducidos. 
Prevalencia de abuso y/o dependencia.

Variables socio-demográficas y patrones de uso de cocaína.

Prevalencias de trastornos psicopatológicos en tres poblaciones: población general, comunidad terapéutica y población ambulatoria.

\section{Procedimiento}

Previo al inicio de la investigación se contó con un miembro del equipo terapéutico responsable del reclutamiento en cada uno de los CTA. Así, esta persona era la encargada de explicar el estudio e invitar a la participación en el mismo a cada paciente que hubiera solicitado en algún momento tratamiento por consumo de cocaína y que cumpliera los criterios de inclusión. Una vez que la persona aceptaba participar, firmaba el consentimiento informado. De esta forma, se derivaba el participante al investigador clínico, y este último citaba al paciente para realizar el proceso completo de evaluación psicopatológica en un mismo día. La duración media de la entrevista fue aproximadamente de 2 a 3 horas. Así, el evaluador se desplazó por los distintos CTA de la provincia de Málaga. Este estudio se llevó a cabo entre 2009 y 2012.

El número de participantes reclutados que inicialmente aceptaron firmar el consentimiento informado fue de 155, de los que 45 participantes desistieron de acudir a la cita. De estos, 15 sujetos no llegaron a firmar el consentimiento informado por presentar criterios de exclusión no detectados en el reclutamiento previo, por ejemplo por alteraciones cognitivas evidentes, o por ser la cocaína sustancia secundaria a la solicitud de tratamiento.

\section{Análisis estadístico}

Los resultados se analizaron mediante el programa estadístico SPSS versión 19.0 (SPSS Inc., an IBM company).

Se emplearon estadísticos descriptivos para caracterizar la muestra que ha participado en el estudio.

Las comparaciones entre grupos o contraste de hipótesis (los que no tienen comorbilidad psicopatológica respecto a los que sí la presentan) se realizaron mediante la prueba Chi Cuadrado $(\chi 2)$ o la fórmula exacta de Fisher en el caso de las variables cualitativas utilizando, y mediante el estadístico $\mathrm{T}$ de Student (t) en el caso de variables dependientes cuantitativas.

Para todas las pruebas de contraste de hipótesis una probabilidad $\mathrm{p}<0,05$ fue considerada como significativa $\mathrm{y}$ $\mathrm{p}<0,01$ fue considerada como altamente significativa. En concordancia con las variables de estudio todas las pruebas se realizaron con la opción de 2 colas (diferencias en ambos sentidos).

Para determinar qué factores están asociados a comorbilidad psicopatológica se utilizó una regresión logística, la prueba de Chi-cuadrado ( $\chi 2)$ de Pearson. En la cual se han incluido covariables como predictoras de presencia o ausencia de comorbilidad psicopatológica en esta población.

\section{Resultados}

\section{Características socio-demográficas}

La descripción de los resultados socio-demográficos de la muestra final de 110 sujetos está descrita en la Tabla 1. El $86,4 \%$ fueron hombres y la media de edad fue de 36,5 años $(\mathrm{DE}=8,8)$. Un 37,3\% eran solteros frente a un $40,9 \%$ que estaban casados o vivían en pareja. Un $69,1 \%$ de la población tenía estudios primarios y un $19,1 \%$ estudios secundarios. Por otro lado, un $58,2 \%$ se encontraba con empleo y un $37,3 \%$ estaba desempleado en el momento de la entrevista. Además, un 46,4\% había tenido algún problema o causa judicial. Finalmente, un 36,4\% de los participantes había recibido un tratamiento psicológico previo en su vida.

Existieron diferencias significativas $(* \mathrm{p}<0,05)$ en la media de edad de los sujetos que no tienen comorbilidad psicopatológica, con 33,9 $(7,9)$ años, de los que sí la presentan, con 38,1 (9,0) años. Por lo tanto, la edad de los pacientes con comorbilidad fue un promedio de 4 años superior en comparación a la población que no presenta comorbilidad psicopatológica.

\section{Patrones de uso de cocaína}

Las variables relacionadas con el consumo de cocaína en esta población de estudio se reflejan también en la Tabla 1. La edad media del primer uso de cocaína para toda la muestra fue de 22,8 (DE=7,2) años. Cabe destacar que existen diferencias, aunque no estadísticamente significativas, entre las dos subpoblaciones contrastadas, siendo la edad de inicio del consumo de cocaína a los 21,9 $(5,8)$ años para los sujetos con comorbilidad psicopatológica y de 24,3 $(8,9)$ años para los sin comorbilidad. Por otro lado, la edad media total del primer uso patológico de cocaína fue de $26(7,5)$ años siguiendo el patrón PRISM de uso patológico. La duración del uso patológico de cocaína en años fue una media de $7,1(7,0)$.

Además, la media total de número de criterios de dependencia según DSM-IV-TR fue de 5,7 (2,2). Finalmente, la abstinencia de cocaína de esta población tuvo una media de $2,7(3,9)$ años.

Existen diferencias altamente significativas $(* * \mathrm{p}<0,01)$ en la media de criterios de dependencia de la población que no presentó comorbilidad psicopatológica, con 4,4 $(2,8)$ criterios, en comparación a los que sí presentaron comorbilidad, $6,5(1,1)$ criterios.

\section{Factores asociados a comorbilidad psicopatológica}

La Tabla 2 representa un análisis de regresión logística en la cual se ha utilizado como variable dependiente la presencia o ausencia de comorbilidad psicopatológica y de covariables predictoras. Los resultados muestran que la variable 
Pedro Araos, Esperanza Vergara-Moragues, María Pedraz, Francisco J. Pavón, Rafael Campos Cloute, Montserrat Calado, Juan Jesús Ruiz, Nuria García-Marchena, Isolde Gornemann, Marta Torrens, Fernando Rodríguez de Fonseca

Tabla 1

Variables socio-demográficas y patrones uso de cocaína en una muestra total ( $N=110$ ) y diferencias entre los que tienen comorbilidad psicopatológica a lo largo de la vida y los que no tienen comorbilidad psicopatológica a lo largo de la vida

\begin{tabular}{|c|c|c|c|c|}
\hline$N=110$ & TOTAL & $\begin{array}{c}\text { Sin comorbilidad psicopatológica } \\
\text { a lo largo vida } \\
N(\%) \\
42(38,2)\end{array}$ & $\begin{array}{c}\text { Con comorbilidad } \\
\text { psicopatológica a lo largo vida } \\
N(\%) \\
68(61,8)\end{array}$ & $p$ \\
\hline $\begin{array}{l}\text { Mujer } \\
\text { Hombre }\end{array}$ & $\begin{array}{l}15(13,6) \\
95(86,4)\end{array}$ & $\begin{array}{c}4(9,5) \\
38(90,5)\end{array}$ & $\begin{array}{l}11(16,2) \\
57(83,8)\end{array}$ & 0,32 \\
\hline Edad [Media (DE)] & $36,5(8,8)$ & $33,9(7,9)$ & $38,1(9,0)$ & $0,01 *$ \\
\hline Situación conyugal actual & $\begin{array}{r}41(37,3) \\
45(40,9) \\
22(20,0) \\
2(1,8) \\
\end{array}$ & $\begin{array}{c}17(40,5) \\
21(50,0) \\
3(7,1) \\
1(2,4)\end{array}$ & $\begin{array}{c}24(35,3) \\
24(35,3) \\
19(27,9) \\
1(1,5)\end{array}$ & 0,14 \\
\hline $\begin{array}{r}\text { Sin estudios } \\
\text { Estudios primarios } \\
\text { Estudios secundarios } \\
\text { Estudios universitarios }\end{array}$ & $\begin{array}{c}2(1,8) \\
76(69,1) \\
21(19,1) \\
11(10,0)\end{array}$ & $\begin{array}{c}- \\
29(69,0) \\
9(21,4) \\
4(9,5)\end{array}$ & $\begin{array}{l}2(2,9) \\
47(69,1) \\
12(17,6) \\
7(10,3)\end{array}$ & 0,69 \\
\hline $\begin{array}{r}\text { Sin empleo } \\
\text { Con empleo } \\
\text { Jubilado/Minusvalía }\end{array}$ & $\begin{array}{c}41(37,3) \\
64(58,2) \\
5(4,5)\end{array}$ & $\begin{array}{c}18(42,9) \\
23(54,8) \\
1(2,4)\end{array}$ & $\begin{array}{c}23(33,8) \\
41(60,3) \\
4(5,9)\end{array}$ & 0,49 \\
\hline $\begin{array}{r}\text { Historia Judicial } \\
\qquad \text { Alguna vez detenido o en prisión } \\
\mathrm{Si} \\
\text { No }\end{array}$ & $\begin{array}{l}51(46,4) \\
59(53,6)\end{array}$ & $\begin{array}{l}16(38,1) \\
26(61,9)\end{array}$ & $\begin{array}{l}35(51,5) \\
33(48,5)\end{array}$ & 0,17 \\
\hline $\begin{array}{r}\text { Alguna vez Tratamiento psicológico } \\
\text { Si } \\
\text { No }\end{array}$ & $\begin{array}{l}40(36,4) \\
70(63,6)\end{array}$ & $\begin{array}{l}11(26,2) \\
31(73,8)\end{array}$ & $\begin{array}{l}29(42,6) \\
39(57,4)\end{array}$ & 0,08 \\
\hline $\begin{array}{l}\text { Edad primer uso patológico cocaína [media (DE)] } \\
\text { Edad primer uso cocaína [media (DE)] }\end{array}$ & $\begin{array}{l}26,0(7,5) \\
22,8(7,2)\end{array}$ & $\begin{array}{l}26,6(8,3) \\
24,3(8,9)\end{array}$ & $\begin{array}{l}25,7(7,0) \\
21,9(5,8)\end{array}$ & $\begin{array}{l}0,55 \\
0,09\end{array}$ \\
\hline $\begin{array}{l}\text { Suma criterios de dependencia cocaína } \\
\text { (severidad) [media (DE)] }\end{array}$ & $5,7(2.2)$ & $4,4(2,8)$ & $6,5(1,1)$ & $<0,01 * *$ \\
\hline $\begin{array}{l}\text { Duración del uso patológico de cocaína } \\
\text { (en años) [media (DE)] } \\
\text { Abstinencia cocaína (en años) } \\
\text { [media (DE)] }\end{array}$ & $\begin{array}{l}7,1(7,0) \\
2,7(3,9)\end{array}$ & $\begin{array}{l}6,9(6,7) \\
2,4(2,9)\end{array}$ & $\begin{array}{l}7,1(7,2) \\
2,9(4,5)\end{array}$ & $\begin{array}{l}0,89 \\
0,54\end{array}$ \\
\hline
\end{tabular}

$\left({ }^{*} p<0,05\right)\left({ }^{* *} p<0,01\right)$ (p Fisher); $N=$ Muestra de sujetos; $D E=$ Desviación estándar.

socio-demográfica edad fue estadísticamente significativa $\left({ }^{*} \mathrm{p}<0,05\right.$; Odds Ratio OR= 1,073; Intervalo de confianza IC $95 \%$ 1,012-1,138) al igual que la edad del primer uso de cocaína que también resultó estadísticamente significativa $(* * \mathrm{p}<0,01$; Odds Ratio OR=2,000; Intervalo de confianza IC $95 \%=1,386-2,888)$.

Tabla 2

Prueba Chi cuadrado ( 2 2) de Pearson, estadístico de bondad de ajuste. Prueba=37,93 p=0,000; correctamente clasificadas: 76,1\%. Las variables incluidas en el análisis de regresión logística son: edad, edad primer uso de cocaína y suma criterios de dependencia cocaína

\begin{tabular}{lcccc}
\hline & OR & B & p & 95\% IC \\
\hline Edad & 1,07 & 0,07 & 0,01 & $1,012-1,138$ \\
\hline Edad primer uso cocaína & 2,00 & 0,69 & 0,00 & $1,386-2,888$ \\
\hline Suma criterios dependencia cocaína & 0,94 & $-0,05$ & 0,07 & $, 883-1,006$ \\
\hline
\end{tabular}

OR= Odds Ratio; $\mathrm{B}=$ Valor Estadístico; $\mathrm{P}=$ Significación $\mathrm{P}$ chi-cuadrado; IC= Índice de confianza. 


\section{Abuso y dependencia de sustancias}

Como se puede observar en la Tabla 3 , un $61,8 \%$ de la población de estudio ha tenido uso de cocaína junto a otra psicopatología a lo largo de la vida. De estos participantes, un $90 \%$ presentó abuso y un $95,6 \%$ dependencia a esta sustancia. Por otro lado, un $38,2 \%$ de la población de estudio no presentó psicopatología asociada al uso de cocaína, de los que un 71,4\% mostró abuso y/o dependencia a lo largo de la vida. Por tanto, un $26,1 \%$ presentó consumo ocasional sin trastorno comorbido asociado a lo largo de la vida y, un $4,4 \%$ presentó un consumo ocasional con otra psicopatología asociada.

Tabla 3

Consumo de cocaína y trastornos por uso de sustancias (TUS) (DSM-IV-TR), más otras psicopatologías. Diagnóstico a lo largo de la vida y último año

\begin{tabular}{|c|c|c|c|c|c|c|}
\hline \multirow{2}{*}{$N=110$} & \multicolumn{3}{|c|}{ Diagnóstico último año } & \multicolumn{3}{|c|}{ Diagnóstico a lo largo vida } \\
\hline & $\begin{array}{c}\text { Sin otra } \\
\text { psicopatología } \\
N(\%) \\
64(58,2)\end{array}$ & $\begin{array}{c}\text { Con otra } \\
\text { psicopatología } \\
N(\%) \\
46(41,8)\end{array}$ & $\mathbf{p}$ & $\begin{array}{c}\text { Sin otra } \\
\text { psicopatología } \\
N(\%) \\
42(38,2)\end{array}$ & $\begin{array}{c}\text { Con otra } \\
\text { psicopatología } \\
N(\%) \\
68(61,8)\end{array}$ & $\mathbf{p}$ \\
\hline \multicolumn{7}{|l|}{ Cocaina } \\
\hline Abuso & $1(1,6)$ & $1(2,2)$ & 1,00 & $30(71,4)$ & $61(90,0)$ & $<0,01^{*}$ \\
\hline Dependencia & $24(37,5)$ & $33(71,7)$ & $<0,01^{* *}$ & $30(71,4)$ & $65(95,6)$ & $<0,01 * *$ \\
\hline Consumo & $8(12,5)$ & $3(6,5)$ & 0,35 & $12(26,1)$ & $3(4,4)$ & $<0,01^{* *}$ \\
\hline \multicolumn{7}{|l|}{ Heroina } \\
\hline Abuso & - & - & - & $2(4,8)$ & $9(13,2)$ & 0,15 \\
\hline Dependencia & - & $2(4,4)$ & 0,09 & $1(2,4)$ & $10(14,7)$ & $<0,03^{*}$ \\
\hline \multicolumn{7}{|l|}{ Alcohol } \\
\hline Abuso & $5(7,8)$ & $5(10,9)$ & 0,73 & $27(64,3)$ & $43(63,2)$ & 0,91 \\
\hline Dependencia & $16(25,0)$ & $15(32,6)$ & 0,38 & $19(45,2)$ & $31(45,6)$ & 0,97 \\
\hline \multicolumn{7}{|l|}{ Cannabis } \\
\hline Abuso & $1(1,6)$ & $2(4,4)$ & 0,95 & $6(14,3)$ & $12(17,6)$ & 0,64 \\
\hline Dependencia & $6(9,4)$ & $1(2,2)$ & 0,12 & $6(14,3)$ & $9(13,2)$ & 0,67 \\
\hline \multicolumn{7}{|l|}{ Benzodiazepinas } \\
\hline Abuso & $2(3,1)$ & $4(8,7)$ & 0,20 & $2(4,8)$ & $7(10,3)$ & 0,30 \\
\hline Dependencia & $2(3,1)$ & $3(6,5)$ & 0,39 & $2(4,8)$ & $7(10,3)$ & 0,30 \\
\hline \multicolumn{7}{|l|}{ Alucinógenos } \\
\hline Abuso & $1(1,6)$ & - & 0,39 & $1(2,4)$ & $4(5,9)$ & 0,39 \\
\hline Dependencia & $1(1,6)$ & - & 0,39 & $1(2,4)$ & $4(5,9)$ & 0,39 \\
\hline \multicolumn{7}{|l|}{ Estimulantes } \\
\hline Abuso & - & - & - & $1(2,4)$ & $2(2,9)$ & 0,86 \\
\hline Dependencia & $1(1,6)$ & - & 0,39 & $1(2,4)$ & $2(2,9)$ & 0,86 \\
\hline \multicolumn{7}{|l|}{ Metadona + } \\
\hline Abuso & - & $1(2,2)$ & 0,23 & $1(2,4)$ & $1(1,5)$ & 0,72 \\
\hline Dependencia & - & $1(2,2)$ & 0,23 & $1(2,4)$ & $1(1,5)$ & 0,72 \\
\hline \multicolumn{7}{|l|}{ Otras Drogas } \\
\hline Abuso & - & - & - & - & - & - \\
\hline Dependencia & - & - & & - & - & - \\
\hline \multicolumn{7}{|c|}{ Policonsumo (tres o más sustancias) } \\
\hline Abuso & $1(1,6)$ & $4(8,7)$ & 0,07 & $6(14,3)$ & $15(22,1)$ & 0,31 \\
\hline Dependencia & $5(7,8)$ & $3(6,5)$ & 0,80 & $5(11,9)$ & $15(22,1)$ & 0,18 \\
\hline
\end{tabular}

$\left({ }^{*} \mathrm{p}<0,05 ;{ }^{* *} \mathrm{p}<0,01\right.$ ) (P de Fisher); $\mathrm{N}=$ Número de sujetos; $\mathrm{P}=$ Probabilidad mediante prueba exacta de Fisher; Consumo = Consumo ocasional de cocaína; + Metadona comprada en la calle.

Para el último año, un 41,8\% de la muestra presentó uso de cocaína concomitante con otra psicopatología. De estos sujetos de estudio, un 2,2\% presentó abuso y un 71,7\% dependencia de cocaína. Por consiguiente, un 6,5\% presentó algún consumo ocasional a esta sustancia el último año. Por otro lado, un 58,2\% de esta población no ha presentado algún trastorno comorbido el último año. De estos participantes, un $1,6 \%$ presentó abuso y un $37,5 \%$ dependencia de cocaína. Por lo tanto, un 12,5\% presentó consumo ocasional a esta sustancia. Debemos consignar que, un 19,5\% de los sujetos diagnosticados con otra psicopatología se encontraba abstinente de la sustancia el último año y, que un 48,4\% de la población sin otra psicopatología se encontraba abstinente para este periodo de tiempo.

Entre las otras sustancias de consumo relacionadas, y en orden descendente, un $63,2 \%$ de la población con comor- 
bilidad presentó abuso de alcohol a lo largo de la vida y un $45,6 \%$ presentó dependencia. Para cannabis, un $17,6 \%$ de abuso y un $14,7 \%$ dependencia. Un $13,2 \%$ presentó abuso y un $14,7 \%$ dependencia de heroína. Un $10,3 \%$ de esta población con comorbilidad presentó abuso y dependencia para benzodiacepinas.

Existieron diferencias altamente significativas $(* * \mathrm{p}<0,01)$ en dependencia de cocaína a lo largo de la vida entre las subpoblaciones con psicopatología y sin psicopatología. Para abuso de cocaína a lo largo de la vida obtuvimos diferencias significativas $(* \mathrm{p}<0,05)$ entre las dos subpoblaciones. Para consumo ocasional de cocaína obtuvimos diferencias altamente significativas entre las dos muestras poblaciones contrastadas a lo largo de la vida $(* * \mathrm{p}<0,01)$. En relación al último año, encontramos diferencias altamente significativas $(* * \mathrm{p}<0,01)$ en dependencia de cocaína entre las subpoblaciones comparadas. Para abstinencia en el último año encontramos diferencias altamente significativas $(* * \mathrm{p}<0,01)$ en las dos subpoblaciones contrastadas.
Para abuso de heroína a lo largo de la vida encontramos diferencias significativas $(* \mathrm{p}<0,05)$ entre las dos subpoblaciones contrastadas.

Debemos decir que para el resto de otras sustancias estudiadas no encontramos diferencias estadísticamente significativas inter grupos.

\section{Comorbilidad psicopatológica}

En relación a la comorbilidad psicopatológica de esta población de estudio $(n=110)$ pudimos detectar que un $61,8 \%$ ha tenido al menos un trastorno del Eje I y/o del Eje II a lo largo de la vida y un 38,2\% no presentó psicopatología para este periodo de tiempo (ver Tabla 4). Los trastornos más prevalentes del Eje I fueron primero, trastornos del estado de ánimo (TEA) con un 34,5\%, de los cuales un 24,5\% fueron TEA inducidos por la sustancia. Segundo, trastornos de ansiedad (TA) con un 22,7\%, de los cuales un 9,1\% fueron TA inducidos. Tercero, trastornos psicóticos (TP) con un $15,5 \%$ de los cuales un 12,7\% son TP inducidos.

Tabla 4

Comorbilidad psicopatológica en el último año y a lo largo de la vida en población total ( $N=110)$. Diagnóstico a lo largo de la vida y diagnóstico último año (Entrevista PRISM-DSM-IV-TR).

\begin{tabular}{|c|c|c|}
\hline \multirow{2}{*}{$\begin{array}{l}\text { N }=110 \\
\text { COMORBILIDAD PSICOPATOLÓGICA }\end{array}$} & \multicolumn{2}{|c|}{ Total $(\%)$} \\
\hline & Diagnóstico último año & Diagnóstico a lo largo vida \\
\hline Alguna co-ocurrencia de comorbilidad psicopatológica (Eje I y/o Eje II) & $46(41,8)$ & $68(61,8)$ \\
\hline EJE I: Trastornos Psicopatológicos & $27(24,5)$ & $57(51,8)$ \\
\hline $\begin{array}{l}\text { Trastorno estado de ánimo } \\
\text { Trastorno estado de ánimo primario } \\
\text { Depresión mayor } \\
\text { Distimia } \\
\text { Ciclotimia } \\
\text { Episodio maniaco } \\
\text { Episodio Hipomaniaco } \\
\text { Trastorno estado de ánimo inducido }\end{array}$ & $\begin{array}{c}19(17,3) \\
9(8,2) \\
8(7,3) \\
1(0,9) \\
- \\
- \\
- \\
10(9,1)\end{array}$ & $\begin{array}{l}38(34,5) \\
18(16,4) \\
16(14,5) \\
2(1,8) \\
1(0,9) \\
1(0,9) \\
2(1,8) \\
27(24,5)\end{array}$ \\
\hline $\begin{array}{l}\text { Trastorno de ansiedad } \\
\text { Trastorno de ansiedad primario } \\
\text { Trastorno de pánico } \\
\text { Trastorno de pánico con agorafobia } \\
\text { Ansiedad generalizada } \\
\text { Agorafobia } \\
\text { Fobia Específica } \\
\text { Fobia Social } \\
\text { Trastorno obsesivo-compulsivo } \\
\text { Trastorno por estrés post-traumático } \\
\text { Trastorno de ansiedad inducida }\end{array}$ & $\begin{array}{c}10(9,1) \\
10(9,1) \\
2(1,8) \\
- \\
- \\
- \\
3(2,7) \\
2(1,8) \\
1(0,9) \\
3(2,7) \\
-\end{array}$ & $\begin{array}{c}25(22,7) \\
18(16,4) \\
2(1,8) \\
- \\
4(3,6) \\
1(0,9) \\
3(2,7) \\
2(1,8) \\
2(1,8) \\
11(10,0) \\
10(9,1)\end{array}$ \\
\hline $\begin{array}{l}\text { Trastorno psicótico } \\
\text { Trastorno psicótico primario } \\
\text { Esquizofrenia } \\
\text { Trastorno esquizofreniforme } \\
\text { Trastorno esquizoafectivo } \\
\text { Trastorno delirante } \\
\text { Trastorno psicótico no especificado } \\
\text { Trastorno estado de ánimo con síntomas psicóticos } \\
\text { Trastorno psicótico inducido }\end{array}$ & $\begin{array}{c}2(1,8) \\
- \\
- \\
- \\
- \\
- \\
- \\
- \\
2(1,8) \\
\end{array}$ & $\begin{array}{l}17(15,5) \\
3(2,7) \\
1(0,9) \\
1(0,9) \\
1(0,9) \\
\quad- \\
- \\
- \\
14(12,7)\end{array}$ \\
\hline $\begin{array}{l}\text { Trastorno de la conducta alimentaria } \\
\text { Anorexia } \\
\text { Bulimia }\end{array}$ & $\begin{array}{c}2(1,8) \\
- \\
2(1,8) \\
\end{array}$ & $\begin{array}{c}3(2,7) \\
- \\
3(2,7)\end{array}$ \\
\hline EJE II: Trastornos de la personalidad & $35(31,8)$ & $41(37,3)$ \\
\hline $\begin{array}{l}\text { Trastorno antisocial de la personalidad (TAP) } \\
\text { Trastorno límite de la personalidad (TLP) }\end{array}$ & $\begin{array}{l}19(17,3) \\
22(20,0)\end{array}$ & $\begin{array}{l}22(20,0) \\
23(20,9)\end{array}$ \\
\hline
\end{tabular}

\%= Porcentaje; Eje I=Trastornos clínicos; Eje II= Trastornos de personalidad. 
Por otro lado, con respecto a los trastornos del Eje II evaluados, se encontró una prevalencia a lo largo de la vida de un $20 \%$ de trastorno antisocial de la personalidad (TAP) y un $20,9 \%$ trastorno límite de la personalidad (TLP).

La prevalencia de comorbilidad psicopatológica para el último año en esta población fue de un 41,8\%, tanto para Eje I y/o Eje II (ver Tabla 4). Para el Eje I tenemos TEA un $17,3 \%$, de los cuales un $9,1 \%$ fue inducido. Para los TA un porcentaje del $9,1 \%$ y para los TP un $1,8 \%$ siendo todos estos inducidos por la sustancia.

Por último en el Eje II tuvimos valores para TAP de un $17,3 \%$ y para TLP de un 20,0\%.

\section{Comparación de psicopatología en poblaciones de Andalucía}

Los resultados obtenidos sobre psicopatologías a lo largo de la vida para PG fueron de un $41,1 \%$, un $61,8 \%$ para CTA y un $65,6 \%$ para CT. Para psicopatologías en el último año detectamos una prevalencia de $28,8 \%$ en $\mathrm{PG}$, un $41,8 \%$ para CTA y un 58,1\% en CT (ver Tabla 5).

Tabla 5

Comparación de psicopatología entre poblaciones, ambulatoria (CTA), general (PG), y comunidad terapéutica (CT) en Andalucía, estimado a lo largo de la vida y último año. Resultados totales

\begin{tabular}{lccc}
\hline $\begin{array}{c}\text { Comparación } \\
\text { psicopatología }\end{array}$ & $\begin{array}{c}\text { CTA } \\
\mathbf{N}=110\end{array}$ & $\begin{array}{c}\text { PG } \\
\mathbf{N}=\mathbf{1 6 0 0}\end{array}$ & $\begin{array}{c}\text { CT } \\
\mathbf{N}=\mathbf{2 2 7}\end{array}$ \\
\hline A lo largo vida & $61,8 \%$ & $42,1 \%$ & $65,6 \%$ \\
\hline Último año & $41,8 \%$ & $28,8 \%$ & $58,1 \%$ \\
\hline
\end{tabular}

$\mathrm{N}=$ Número de sujetos

\section{Discusión}

La coexistencia entre TUS y otras psicopatologías se han estudiado en diferentes contextos terapéuticos y para distintas sustancias de abuso, (Kessler et al., 2005; Kidorf et al., 2004).

De acuerdo con nuestro objetivo principal, la prevalencia de comorbilidad psicopatológica de este estudio a lo largo de la vida ha sido alta $(61,8 \%)$ en un rango parecido a los estudios realizados sobre distintos tipos de muestras con personas consumidoras de cocaína, tanto en tratamiento ambulatorio como de comunidades terapéuticas, así como en población no clínica (Adamson, Tood, Sellman, Huriwai y Porter, 2006; Arias et al., 2012; Herrero et al., 2008; Levín, Evans, Vosburg, Horton y Brooks, 2004; Mardsen, Gossop, Stewart, Rolfe y Farrell, 2000; Swendsen et al., 2010; Tortajada et al.,2012). Tanto los estudios llevados a cabo en población general como los de muestras clínicas refieren que la prevalencia de la concurrencia de trastornos por consumo de sustancias y otras psicopatologías es elevada, situándose entre el 15-80\% (Nocon, Bergé, Astals, Martín-Santos y Torrens, 2007; Rodríguez-Llera et al., 2006;). A la luz de los resultados, y de acuerdo con otros trabajos, nos parece esencial tratar a estos pacientes integradamente, es decir, que se garantice el abordaje de la adicción y otras psicopatologías de forma conjunta. Sería necesaria la creación de modelos de atención integrados desde una perspectiva individualizada y flexible (Sacks, Banks, McKendrik y Sacks, 2008).

$\mathrm{Si}$ analizamos la comorbilidad psicopatológica encontramos que, primero, los (TEA) inducidos a lo largo de la vida son más prevalentes que los TEA primarios. Por otra parte, y al contrario de estos resultados obtenidos, podemos mencionar estudios de comorbilidad psicopatológica en consumidores de drogas que obtuvieron resultados contrarios a los nuestros para TEA, siendo en esos trabajos más prevalentes los TEA primarios que los TEA inducidos (Rodríguez-Llera et al., 2006; Herrero et al., 2007). Debemos considerar que estos estudios se han realizado en distintos tipos de muestras y en algún caso con droga que no es la cocaína. En cualquier caso, la alta prevalencia de TEA es significativa en esta población y ello podría ser relevante para el proceso terapéutico. Sería interesante tomar en cuenta la variable abuso y dependencia de alcohol en este tipo de poblaciones consumidoras de cocaína, por ser la sustancia de abuso más prevalente de este tipo de sujetos y muy ligada con la clínica afectiva inducida (Magidson, Wang, Lejuez, Iza y Blanco, 2013).

Segundo, en nuestro estudio y respecto a los (TA) han sido más prevalentes los primarios que los inducidos para los diagnósticos a lo largo de la vida. Especial interés nos despierta la alta prevalencia para esta muestra de trastorno por estrés post traumático (TEP) (10\%), algunos estudios hablan de prevalencias aún más altas en pacientes con trastornos por uso de sustancias y TEP, entre un $25 \%$ a $42 \%$ (Brady, Back y Coffey, 2004; Jacobson, Southwick y Kosten, 2001) podríamos especular que la alta prevalencia en TEP está relacionada con la vivencia de sucesos ambientales traumáticos (Back, Dansky, Carroll, Foa y Brady, 2001; Najavist et al., 2003) la presencia de un TEP está asociado a una mayor gravedad de la psicopatología y un peor pronóstico (Back et al., 2001). Creemos que en nuestra población de estudio podrían influir factores socio-demográficos que precipitarían la probabilidad de desarrollar un TEP.

Tercero, la prevalencia de trastornos psicóticos (TP) encontrada en esta población consumidora de cocaína en tratamiento ambulatorio es especialmente relevante siendo en su mayoría, trastornos inducidos. Pensamos en la necesidad de identificar adecuadamente estos trastornos sean primarios o inducidos, y así hacer un seguimiento a esta población diagnosticada y monitorizar la evolución de la sintomatología durante todo el proceso terapéutico llevado a cabo en estos centros de tratamiento. 
Por último, la prevalencia de trastorno antisocial de la personalidad (TAP) es de un $20 \%$ y de trastorno límite de la personalidad (TLP) de un $21 \%$ a lo largo de la vida respectivamente, en concordancia con otros estudios internacionales que relacionan consumo de cocaína y trastornos de personalidad (Chen et al., 2011; Grant et al., 2004). Si se realiza una revisión de los estudios que valoran la prevalencia de trastornos de personalidad en población drogodependiente se observa que el porcentaje es muy dispar entre un 36,7\% hasta el 97\% (Calsyn y Saxon, 1990; Fernandez-Montalvo et al., 2003). Así que probablemente, nuestros resultados son menores debido a que la PRISM valora sólo dos trastornos de personalidad, por tanto, consideramos necesario agregar otras baterías diagnósticas de personalidad, con la finalidad de tener una panorámica dimensional de estos trastornos. Favoreciendo la clasificación, evaluación, explicación y conceptualización de estos trastornos.

Por otro lado, quisimos conocer el perfil de nuestra muestra. Así nos indica que es una población particularmente masculina con un bajo nivel educativo, un alto grado de desempleo y problemas judiciales recurrentes.

Con respecto, a los patrones de uso de cocaína, primero, encontramos que la edad del primer uso para esta sustancia es similar a la media europea de inicio de consumo (OEDT, 2012).Por tanto, la edad media total del primer uso de cocaína de nuestra población podría concordar con los datos de otros estudios epidemiológicos internacionales (Wagner y Anthony, 2006; Van Etten y Anthony, 1999). Segundo, los pacientes que se diagnosticaron con comorbilidad psicopatológica comenzaron a usar de la cocaína en una media de dos años antes que los sujetos que no presentaron comorbilidad. Por último, la transición del uso al abuso/dependencia en nuestra población de estudio está en una media de 25 años en hombres, que podría relacionarse con estudios epidemiológicos que hablan de un comienzo de uso patológico entre los 23-25 años (Wagner y Anthony, 2002; Vega et al., 2002).

La prevalencia de abuso y dependencia de cocaína que se ha encontrado ha sido más elevada en la población que presenta comorbilidad psicopatológica. Por tanto, sería importante seguir evaluando la relación entre comorbilidad de trastornos, primarios e inducidos, y la severidad en el consumo (abuso/dependencia). Ya que, podríamos intuir que la severidad en el consumo podría estar relacionada con esta comorbilidad.

Nos parece importante referirnos a esta transición de uso hacia abuso/dependencia, la cual nos podría proporcionar información acerca de la etiología y curso de la severidad de la adicción, pensamos que sería una guía útil tanto para profesionales a nivel preventivo como a nivel clínico la identificación de pacientes con mayor riesgo de desarrollar dependencia. Creemos importante seguir investigando sobre este tema.
Otro tema de interés, es el de la abstinencia en esta población, se ha demostrado estadísticamente en este estudio que, los sujetos con comorbilidad psicopatológica responden peor a la mantención de la abstinencia comparada con la subpoblación que no presenta comorbilidad en el último año. Esta relación sería interesante de tomar en cuenta.

Es importante discutir los resultados significativos de los análisis de regresión logística efectuados, en el cual aparecen como predictores de comorbilidad psicopatológica, la edad y la edad del primer uso de cocaína. Nos parece interesante relacionarlo con otro resultado de este estudio, mencionado anteriormente, aunque no son datos significativos, nuestra población con comorbilidad psicopatológica comenzó a utilizar la cocaína en una media de 2 años antes que los sujetos sin comorbilidad, en este caso, los participantes con comorbilidad tienen una media de edad de inicio de consumo similar a otros estudios epidemiológicos relacionados con consumo de cocaína (Lopez-Quintero et al., 2011). Esto no quiere decir que no tengamos en un futuro en cuenta otras variables que podrían ser importantes en el proceso adictivo.

Por último, se analizaron las diferentes prevalencias con otras poblaciones de estudio, comparando estas muestras tanto a lo largo de la vida como en el último año. Encontramos desigual porcentaje entre PG y las dos poblaciones clínicas estudiadas CTA y CT. Como cabía esperar, el estudio realizado en PG muestra un menor porcentaje de psicopatología en comparación con las otras dos poblaciones, tanto a lo largo de la vida como en el último año, lo que estaría respaldado por otros estudios comparativos de comorbilidad psicopatológica (Gual, 2007; Ziedonis, Rayford, Bryant y Rounsaville, 1994). Se debe aclarar que nuestro estudio tiene criterios de inclusión diferentes que la CT, esto podría marcar las diferencias de prevalencias de trastornos en el último año entre las dos poblaciones clínicas. Podemos agregar, que nuestra población ambulatoria es usuaria activa del dispositivo terapéutico con una media de dos años utilizando el recurso, llevando un promedio de dos años de abstinencia controlada, en cambio los pacientes ingresados en CT en su mayoría son nuevos ingresos que cumplieron criterios diagnósticos de dependencia de cocaína en el último año. Otro punto a tener en cuenta, es que la PG fue valorada con otro instrumento de medida, en relación a las otras dos poblaciones de estudio. Por este motivo, podría limitarse la adecuada comparación entre PG y las otras dos investigaciones. La similitud de resultados a lo largo de la vida entre CTA y CT se podrían deber primero, a las características socio-culturales de la población de Andalucía y, segundo, a que ambas investigaciones se han realizado con el mismo instrumento de medida, la PRISM. Por ejemplo, muchos pacientes que entran en CTA han tenido estancias en CT. De esta manera, como se ha explicado en la parte metodológica, las CTA son la puerta de entrada a los otros dispositivos terapéuticos incluidos la CT. 
Se encuentran una serie de limitaciones en nuestro estudio de población ambulatoria, primero, señalar el sesgo de recuerdo que tiene el sujeto entrevistado. Segundo el tamaño de nuestra muestra, aunque relevante desde un punto de vista clínico, es relativamente pequeño desde una perspectiva estadística y, tercero, mencionar la dificultad de encontrar población femenina entre los usuarios de este dispositivo terapéutico. Por último, al interpretar todos nuestros datos, debemos tener en cuenta que nuestro estudio se realiza en un contexto muy concreto y por tanto habría que tener precaución a la hora de extrapolar los datos a otras poblaciones.

A pesar de ello, hasta lo que conocemos es el primer estudio nacional y autonómico que utiliza una entrevista específica (PRISM) para estudiar la comorbilidad psicopatológica en consumidores de cocaína en población ambulatoria.

Por último, resaltar el valor que podría tener el diagnóstico de comorbilidad psicopatológica en correspondencia al éxito terapéutico y la necesidad de diseñar estrategias para alcanzar mejorar la intervención en los dispositivos asistenciales (Sacks et al., 2008).

Las perspectivas futuras a considerar pasan por continuar con este tipo de estudios de caracterización fenotípica en población andaluza. Primero, desarrollar un estudio longitudinal que permita comprobar la evolución de síntomas psicopatológicos y de severidad en la adicción. Segundo, ampliar la búsqueda de población femenina que cumpla criterios de inclusión, con la finalidad de hacer comparaciones de género y por último, estimar la realidad de una población usuaria de cocaína que está infravalorada diagnósticamente con el objetivo de diseñar tratamientos específicos para población con comorbilidad psicopatológica.

\section{Reconocimientos}

Agradecemos a todos los participantes de este estudio de los distintos Centros de Tratamiento Ambulatorio de la provincia de Málaga, CTA-Mijas-Costa, Centro de Adicciones Alternativa 2- Fuengirola, CTA-Málaga (Carretera de Cádiz) y CTA-Vélez-Málaga, y a cada uno de los reclutadores de estos mismos centros que han hecho posible la realización de este proyecto.

\section{Financiación}

Este artículo ha sido financiado por el Plan Nacional sobre Drogas (PND 049/2009) y la Red de Trastornos Adictivos (RTA), proyectos RD06/0001 y RD012/0028, Instituto de Salud Carlos III, Ministerio de Economía y Competitividad.

\section{Conflictos de intereses}

No existen conflictos de intereses.

\section{Referencias}

Adamson, S. J., Tood, F. C., Sellman, J. D., Huriwai, T. y Porter, J. (2006). Coexisting psychiatric disorders in a New Zealand outpatient alcohol and other drug clinical population. Australian $\mathcal{E}$ New Zealand Journal of Psychiatric, 40, 164-170.

Arias, F., Szerman, N., Vega, P., Mesías, B., Basurte, I., Morant, C.,... Babin, F. (2012). Abuso o dependencia a la cocaína y otros trastornos psiquiátricos. Estudio Madrid sobre la prevalencia de la patología dual. Revista de Psiquiatría y Salud Mental, S1888-9891 (12)00185-1. doi: 10.1016/j.rpsm.2012.09.002.

Back, S. E., Dansky, B. S., Carroll, K. M., Foa, E. B. y Brady, K. T. (2001). Exposure therapy in the treatment of PTSD among cocaine-dependent individuals: description of procedures. Journal Substance Abuse Treatment, 21, 35-45.

Brady, K. T., Back, S. E. y Coffey, S. F. (2004). Substance abuse and posttraumatic stress disorder. Current Directions in Psychological Science, 13, 206-209.

Calsyn, D. A. y Saxon, A. J. (1990). Personality disorder subtypes among cocaine and opioids addicts using the Millon Clinical Multiaxial Inventory. International Journal of the Addictions, 25, 1037-1049.

Chen, K. W., Banducci, A., Guller, L., Macatee, R. J., Lavelle, A. Daughters, S. y Lejuez, C. W. (2011). An Examination of Psychiatric Comorbidities as a Function of Gender and Substance Type within an Inpatient Substance Use Treatment Program. Drug and Alcohol Dependence, $118(2$ 3):92-99. doi:10.1016/j.drugalcdep.2011.03.003.

Dutra, L., Stathopoulou, G., Basden, S., Leyro, T., Powers, M. y Otto, M. (2008). A meta-analytic review of psychosocial interventions for substance use disorders. American Journal of Psychiatry, 165, 179-187. doi: 10.1176/appi. ajp.2007.06111851.

Fernández-Montalvo, J., Lorea, I., López-Goñi, J. J. y Landa, N. (2003). Trastornos de personalidad en adictos a cocaína: Un estudio piloto. Análisis y Modificación de Conducta, 29, 79-98.

Fiedler, K. K., Kim, N., Kondo, D. G. y Renshaw, P. F. (2012). Cocaine use in the past year is associated with altitude of residence. Journal of Addiction Medicine, 6, 166-71. doi: 10.1097/ADM.0b013e31824b6c62.

Goldsmith, R. J. (1999). Overview of psychiatric comorbidity: practical and theoretic considerations. The Psychiatric Clinics of North America, 22, 331-349.

Gornemann, I. (2002). Prevalencia del trastorno mental en la población de Andalucía. Tesis Doctoral. Granada: Universidad de Granada.

Grant, B. F., Hasin, D. S., Stinson, F. S., Dawson, D. A., Chou, S. P., Ruan, W. J. y Pickering, R. P. (2004). Prevalence correlates, and disability of personality disorders in the United States: results from the national epidemiologic survey on alcohol and related conditions. Journal of Clinical Psychiatry, 65, 948-958. 
Gual, A. (2007). Dual Diagnosis in Spain. Drug and Alcohol Review, 26, 65-71.

Hasin, D., Trautman, K., Miele, G., Samet, S., Smith, M. y Endicott, J. (1996). Psychiatric Research Interview for Substance and Mental Disorders (PRISM): Reliability for substance abusers. American Journal of Psychiatry, 153:1195-1201.

Herrero, M. J., Domingo-Salvany, A., Torrens, M., Brugal, M. T. y ITINERE Investigators. (2007). Psychiatric comorbidity in young cocaine users: induced versus independent disorders. Addiction, 103, 284-293.doi: 10.1111/j.1360-0443.2007.02076.x.

Herrero, M. J., Domingo-Salvany, A., Brugal, M. T., Torrens, M., Gutierrez, F. y the ITINERE Investigators. (2008). Personality Profile in Young Current Regular Users of Cocaine. Substance Use E Misuse, 43, 1378-1394. doi:10.1080/10826080801922652.

Jacobsen, L. K., Southwick, S. M. y Kosten, T. R. (2001). Substance use disorders in patients with posttraumatic stress disorder among substance users from the general population. American Journal of Psychiatry, 158, 11841190.

Karila, L., Petit, A., Lowenstein, W. y Reynaud, M. (2012). Diagnosis and consequences of cocaine addiction. Current Medical Chemistry, 19, 5612-5618.

Karila, L., Zarmdini, R., Petit, A., Lafaye, G, Lowenstein, W. y Reynaud, M. (2013). Cocaine addiction: Current data for clinician. Presse Medicale, 30 . Pii S075-4982 (13)00566. doi: 10.1016/j.lpm.2013.01.069.

Kessler, R. C., Chiu, W. T., Demler, O., Merikangas, K. R. y Walters E. E. (2005). Prevalence, severity, and comorbidity of the 12-month DSM-IV disorders in the National Comorbidity Survey Replication. Archives of General Psychiatry, 62, 617-27.

Kidorf, M., Disney, E. R., King, V. L., Neufeld, K., Beilenson, P. L. y Brooner, R. K. (2004). Prevalence of psychiatric and substance use disorders in opioid abusers in a community syringe exchange program. Drug and Alcohol Dependence, 74, 115-122.

Kleinman, P. H., Miller, A. B., Millman, R. B., Woody, G. E., Todd, T., Kemp, J. y Lipton, D.S. (1990) Psychopathology among cocaine abusers entering treatment. Journal of Nervous and Mental Disease, 178, 442-447.

Lopez-Quintero, C, Pérez de los Cobos, J, Hasin, D. S, Okuda, M, Wang, S, Grant, B. F. y Blanco C. (2011). Probability and predictors of transition from first use to dependence on nicotine, alcohol, cannabis and cocaine: results of the National Epidemiologic Survey on Alcohol and Related Conditions (NESARC). Drug and Alcohol Dependence, 1; 115, 120-30. doi: 10.1016/j.drugalcdep.2010.11.004.

Levín, F. R., Evans, S. M., Vosburg, S. K., Horton, T., Brooks, D. y Ng, J. (2004). Impact of attention-deficit hyperactivity disorder and other psychopathology on treatment retention among cocaine abusers in therapeutic community. Addictive Behaviors, 29, 1875-1882.

Magidson, J. F., Wang, S., Lejuez, C. W., Iza, M. y Blanco, C. (2013). Prospective study of substance-induced and independent major depressive disorder among individuals with substance use disorders in a nationally representative simple. Depression and Anxiety, 30, 538-45. doi. 10.1002/da.22122.

Mardsen, J., Gossop, M., Stewart, D., Rolfe, A. y Farrell, M. (2000). Psychiatric symptoms among clients seeking treatment for drug dependence. Intake data from the National Treatment Outcome Research Study. British Journal of Psychiatry, 176, 285-289.

Morgello, S., Holzer, C. E. III, Ryan, E., Young, C., Naseer, M., Castellon S. A.,... Singer, E. J. (2006). Interrater reliability of the Psychiatric Research Interview for Substance and Mental Disorders in an HIV-infected cohort: experience of the National NeuroAIDS Tissue Consortium. International Journal of Methods in Psychiatric Research, 15, 131-138.

Najavist, L. M., Runkel, R., Neuner, C., Frank, A., Thase, M., Crits-Cristoph, P.y Blaine, J. (2003). Rates and symptoms of PTSD among cocaine dependent patients. Journal of Studies on Alcohol, 64, 601-606.

Nocon, A., Bergé, D., Astals, M., Martín-Santos, R. y Torrens, M. (2007) Dual diagnosis in a patient drug abuse detoxification unit. European Addiction Research, 13, 192 200.

Observatorio Europeo de las Drogas y las Toxicomanías (OEDT). (2012). Informe anual 2012: el problema de la drogodependencia en Europa. Luxemburgo: Oficina de Publicaciones de la Unión Europea. doi:10.2810/64802

Office of National Drug Control Policy (ONDCP). (2012). Report 2012: National Drug Control Strategy. Washington, D. C.: Executive office of the President of the United States. The White House.

Pavarin, R. M., (2006).Substance use and related problems: a study on the abuse of recreational and not recreational drugs in Northern Italy. Annali dell'Istituto Superiore di Sanita, 42, 477-484.

Rivera, B., Casal, B., Currais, L. y Rungo, P. (2012). Valoración del impacto económico del consumo de drogas ilegales en Galicia desde una perspectiva social. Presupuesto y Gasto Público, 66, 109-126.

Rodriguez-Llera, M. C., Domingo-Salvany, A., Brugal, M. T., Silva, T. C., Sánchez-Niubo, A. y Torrens, M. (2006). Psychiatric comorbidity in young heroin users. Drug and Alcohol Dependence, 84, 48-55.

Rounsaville, B. J. Treatment of cocaine dependence and depression. (2004). Biological Psychiatry, 56, 803-809.

Ruffles, G. Emergency care for Young people who have used cocaine. (2009). Emergency Nurse, 17, 34-36.

Sacks, S., Banks, S., McKendrik, K. y Sacks, J. Y. (2008). Modified therapeutic community for co-occurring dis- 
orders: a summary of four studies. Journal of Substance Abuse Treatment, 34, 112-122

Swendsen, J., Conway, K. P., Degenhardt, L., Glantz, M., Jin, R., Merikangas, K. R. y Kessler, R. C. (2010). Mental disorders as risk factors for substance use, abuse and dependence: results from the 10 -year follow-up of the National Comorbidity Survey. Addiction, 105, 117-128. doi:10.1111/j.1360-0443.2010.02902.x.

Schuckit, M. A. (2006). Comorbidity between substance use disorders and psychiatric conditions. Addiction, 101, 76-88.

Tortajada, S., Herrero, M. J., Domingo-Salvany, A., Molist, G., Barrio G., de la Fuente, L. y Grupo de Investigación ITINERE. Morbilidad psiquiátrica en consumidores de cocaína y heroína reclutados en la comunidad. (2012). Adicciones, 24, 201-210.

Torrens, M., Gilchrist, G., Domingo-Salvany y the PsyCoBarcelona Group. (2011). Psychiatric comorbidity in illicit drug users: Substance-induced versus independent disorders. Drug and Alcohol Dependence, 113, 147-156. doi:10.1016/j.drugalcdep.2010.07.013.

Torrens, M., Serrano D., Astals, M., Perez-Domínguez, G. y Martin-Santos, R. (2004). Diagnosing comorbid psychiatric disorders in substance abusers: validity of the Spanish versions of the Psychiatric Research Interview for Substance and Mental Disorders and the Structured Clinical Interview for DSM-IV. American Journal of Psychiatry; 161, 1231-1237.

Van Etten, M. L. y Anthony, J. C. (1999). Comparative epidemiology of initial drugs opportunities and transitions to first use: cannabis, cocaine, hallucinogens and heroin. Drug and Alcohol Dependence, 54, 117-125.
Vega, W. A., Aguilar-Gaxiola, S., Andrade, L., Bijl, R., Borges, G., Caraveo-Anduaga, J. J.,...Wittchen, H. U.(2002). Prevalence and age of onset for drug use in seven international sites: results from the international consortium of psychiatric epidemiology. Drug and Alcohol Dependence, 68, 285-297.

Vergara-Moragues, E., González-Saíz, F., Lozano, O.M., Betanzos, P., Fernández, F., Bilbao-Acebos, I.,...Verdejo, A. (2012). Psychiatric comorbidity in cocaine users treated in therapeutic community. Substance induced versus independent disorders. Psychiatry Research, 30, 734-41. doi: 10.1016/j.psychres.2012.07.043.

Wagner, F. A. y Anthony, J. C. (2002). From first drug use to drug dependence: developmental periods of risk of dependence upon cannabis, cocaine and alcohol. Neuropsychopharmacology, 26, 479-488.

Wagner, F A. y Anthony, J. C. (2006). Male-Female differences in the risk of progression from first use to dependence upon cannabis, cocaine, and alcohol. Drug and Alcohol Dependence, 86, 191-198.

Weiser M., Reichenberg A., Rabinowitz J., Kaplan Z., Caspi A., Yasvizky R.,...Davidson, M. (2003). Self-reported drug abuse in male adolescents with behavioral disturbances, and follow-up for future schizophrenia. Biological Psychiatry, 54, 655-60.

Ziedonis, D. M, Rayford, B. S., Bryant, K. J. y Rounsaville, B. J. (1994). Psychiatric comorbidity in white and African-American cocaine addicts seeking substance abuse treatment. Hospital E̋ Community Psychiatry, 45, 43-49. 